\title{
Bayesian full-waveform inversion of tube waves to estimate fracture aperture and compliance
}

\author{
Jürg Hunziker ${ }^{1}$, Andrew Greenwood ${ }^{1,2}$, Shohei Minato ${ }^{3}$, Nicolás Daniel Barbosa ${ }^{4}$, Eva Caspari ${ }^{1,2}$, and \\ Klaus Holliger ${ }^{1,5}$ \\ ${ }^{1}$ Applied and Environmental Geophysics Group, Institute of Earth Sciences, University of Lausanne, Lausanne, Switzerland \\ ${ }^{2}$ Chair of Applied Geophysics, Montanuniversität Leoben, Leoben, Austria \\ ${ }^{3}$ Faculty of Civil Engineering and Geosciences, Department of Geoscience and Engineering, \\ Technical University Delft, Delft, the Netherlands \\ ${ }^{4}$ Department of Earth Sciences, University of Geneva, Geneva, Switzerland \\ ${ }^{5}$ School of Earth Sciences, Zhejiang University, Hangzhou, China
}

Correspondence: Jürg Hunziker (jurg.hunziker@unil.ch)

Received: 28 November 2019 - Discussion started: 16 December 2019

Revised: 12 March 2020 - Accepted: 24 March 2020 - Published: 29 April 2020

\begin{abstract}
The hydraulic and mechanical characterization of fractures is crucial for a wide range of pertinent applications, such as geothermal energy production, hydrocarbon exploration, $\mathrm{CO}_{2}$ sequestration, and nuclear waste disposal. Direct hydraulic and mechanical testing of individual fractures along boreholes does, however, tend to be slow and cumbersome. To alleviate this problem, we propose to estimate the effective hydraulic aperture and the mechanical compliance of isolated fractures intersecting a borehole through a Bayesian Markov chain Monte Carlo (MCMC) inversion of full-waveform tube-wave data recorded in a vertical seismic profiling (VSP) setting. The solution of the corresponding forward problem is based on a recently developed semianalytical solution. This inversion approach has been tested for and verified on a wide range of synthetic scenarios. Here, we present the results of its application to observed hydrophone VSP data acquired along a borehole in the underground Grimsel Test Site in the central Swiss Alps. While the results are consistent with the corresponding evidence from televiewer data and exemplarily illustrate the advantages of using a computationally expensive stochastic, instead of a deterministic inversion approach, they also reveal the inherent limitation of the underlying semi-analytical forward solver.
\end{abstract}

\section{Introduction}

Tube waves are interface waves propagating along the borehole wall. They are sometimes also referred to as Stoneley waves, but, as Daley et al. (2003) point out, Scholte waves might be more appropriate as tube waves propagate along a solid-liquid interface. Primary sources of tube waves are ground roll passing over the well head (e.g., Hardage, 1981) or body waves encountering open fractures intersecting the borehole (e.g., Minato and Ghose, 2017; Greenwood et al., 2019b). Secondary sources are the borehole tool itself (e.g., Hardage, 1981) as well as changes in borehole radius or in acoustic impedance within the borehole annulus (e.g., Greenwood et al., 2019b).

Various modeling approaches have been proposed to study the properties of tube waves. A number of analytical techniques to calculate the tube-wave velocity (e.g., Chang et al., 1988; Norris, 1990) as well as semi-analytical methods to simulate complete waveforms (e.g., Cheng and Toksöz, 1981) have been published. To properly reproduce the effects of the borehole environment in finite-difference simulations, one needs a grid refinement in the immediate vicinity of the borehole (e.g., Falk et al., 1996; Sidler et al., 2013). Alternatively, a combination of a semi-analytical solution to model the borehole and a finite-difference approach to model the heterogeneous embedding background medium can be employed (e.g., Kurkjian et al., 1994). 
As tube waves propagate along the borehole, no geometrical spreading occurs, and, therefore, tube waves are much less attenuated than body waves and retain high amplitudes even at large distances from the source. Thus, if vertical seismic profiling (VSP) data are recorded with pressure sensors, such as hydrophones, tube waves tend to pose a problem as they cover body-wave reflections (e.g., Greenwood et al., 2019a, b). Without suppression or removal of the tube waves, reflections in hydrophone VSP data can, in general, only be interpreted at large source-receiver distances and then only before the tube waves and their reverberations arrive (Coates, 1998). Suppression of tube waves during data acquisition is discussed, for example, by Hardage (1981), Daley et al. (2003), and Greenwood et al. (2019b), amongst others. Methods to remove tube waves during data processing are proposed, for example, by Hardage (1981), Herman et al. (2000), and Greenwood et al. (2019a).

Here, we do not aim at suppressing or removing tube waves but rather consider them as signals containing valuable information for characterizing hydraulically open fractures along the borehole, which is important for a wide variety of applications, such as groundwater management, geothermal energy production, hydrocarbon exploration, $\mathrm{CO}_{2}$ sequestration, and nuclear waste disposal. If a tube wave is generated at a fracture due to an incident $\mathrm{P}$ wave, the amplitude ratio of the two wave types can be used to estimate fracture compliances (e.g., Bakku et al., 2013) or fracture permeability (e.g., Hardin and Toksöz, 1985; Li et al., 1994), while the amplitude ratio of the P-wave-induced tube waves to the Swave-induced tube waves can be inverted for the orientation of fractures (e.g., Lee and Toksöz, 1995). The algorithm of Hornby et al. (1989) uses the arrival times of reflected tube waves to invert for the locations of permeable fractures and the reflectivity of tube waves to estimate the effective aperture of fractures. In the field of seismoelectrics, Zhu et al. (2008) showed that tube waves create electromagnetic waves when encountering fractures, which also have the potential to be used for fracture characterization.

The above methods do, however, require extensive manual conditioning of the data, like amplitude picking or timegating of events. Furthermore, they are unable to provide an estimate of uncertainty and/or to identify multiple solutions that are equally likely. The objective of this work is to alleviate these limitations by providing an algorithm that considers the entire wave field for characterizing fractures in terms of their hydraulic apertures and mechanical compliances as well as the associated uncertainties with a minimal amount of human interaction. To this end, we propose a Bayesian full-waveform inversion approach in combination with a recent semi-analytical approach (Minato and Ghose, 2017; Minato et al., 2017) as an efficient and robust forward solver. The proposed algorithm uses as input the complete $\mathrm{P}$ - and tube-wave fields with minimal preprocessing to invert for the effective hydraulic fracture aperture, the mechanical fracture compliance, the bulk and shear modulus of the background rock, and some auxiliary parameters. We use a stochastic inversion algorithm in order (1) to obtain an entire ensemble of solutions, which, in turn, provides a measure of uncertainty, and (2) to account for the strong nonlinearity of the problem and to avoid getting stuck in local minima. We first present our stochastic full-waveform inversion approach, followed by a synthetic example and an application to field data from the underground Grimsel Test Site (https://www.grimsel.com, last access: 21 April 2020) in Switzerland and a subsequent discussion of the results.

\section{Method}

The goal of our stochastic inversion approach is to estimate the posterior probability density function (PDF) $p(\mathbf{m} \mid \mathbf{d})$, which in stochastic terms describes the adequacy of a model $\mathbf{m}$ given the observed data $\mathbf{d}$. We do this by relying on the following approximation of Bayes' theorem (Bayes, 1763):

$p(\mathbf{m} \mid \mathbf{d}) \propto p(\mathbf{m}) L(\mathbf{m} \mid \mathbf{d})$,

where $p(\mathbf{m})$ is the prior PDF describing any a priori knowledge we have about the model parameters and $L(\mathbf{m} \mid \mathbf{d})$ is the likelihood quantifying how well a model $\mathbf{m}$ explains the data d. Following Tarantola (2005), we define the likelihood as

$L(\mathbf{m} \mid \mathbf{d})=\frac{1}{(2 \pi)^{D / 2} \sigma_{\mathrm{e}}^{D}} \exp \left(-\frac{1}{2 \sigma_{\mathrm{e}}^{2}} \sum_{j=1}^{D}\left(G_{j}(\mathbf{m})-d_{j}\right)^{2}\right)$,

where $D$ and $\sigma_{\mathrm{e}}$ are the amount of data points and the standard deviation of the data-error, respectively. The forward operator $G$ calculates synthetic data $\mathbf{d}^{\text {syn }}$ based on a model m:

$\mathbf{d}^{\text {syn }}=G(\mathbf{m})$.

We use a novel semi-analytical algorithm for $G$, which evaluates the Green's function analytically in the frequencyspace domain for a zero-offset VSP setting (Minato and Ghose, 2017). This is done in parallel for a limited number of individual frequencies. Then, Green's functions for the complete frequency band are obtained by spline interpolation. The frequencies for which Green's functions are actually calculated are selected such that the maximum error caused by the interpolation (i.e., the difference between an interpolated and a fully calculated dataset) is 2 orders of magnitude smaller than the largest value in the dataset. After multiplication with the Fourier transform of the source wavelet and a subsequent inverse Fourier transformation, we obtain the full-waveform signals in the time-space domain.

In the considered forward operator $G$, seismic tube waves are generated and scattered at fractures characterized by their static apertures $L_{0}$ and compliances $Z$. A tube wave is generated when a $\mathrm{P}$ wave hits a fluid-filled fracture intersecting the borehole, as the fracture is compressed and fluid is injected 
into the borehole. We describe this process in the frequency domain for a horizontal fracture with the tube-wave generation potential $\phi_{\mathrm{g}}$ (Minato and Ghose, 2017):

$\phi_{\mathrm{g}}(z)=\sum_{i=1}^{N} \frac{2}{\rho_{\mathrm{f}} c_{\mathrm{T}}} \frac{p_{\mathrm{t}}^{(i)}}{p_{\mathrm{inc}}^{(i)}} \delta\left(z-z_{i}\right)$,

where $N$ is the number of fractures in the medium, $\rho_{\mathrm{f}}$ the density of the fluid, and $\delta$ the Dirac delta function. Depth is denoted by $z$, and sub- or superscripts $i$ refer to the $i$ th fracture. Note that this formulation requires the depth vector $z$ to explicitly sample the depth levels of all prevailing fractures. Therefore, the sampling along $z$ determines the minimal distance between two adjacent fractures that can be resolved. The tube-wave velocity $c_{\mathrm{T}}$ is given by (White, 1983)

$c_{\mathrm{T}}={\sqrt{\rho_{\mathrm{f}}\left(\frac{1}{K_{\mathrm{f}}}+\frac{1}{\mu}\right)}}^{-1}$,

with $K_{\mathrm{f}}$ and $\mu$ being the fluid bulk modulus and the shear modulus of the formation, respectively. The pressure fields of the tube wave $p_{\mathrm{t}}^{(i)}$ and the incoming $\mathrm{P}$ wave $p_{\text {inc }}^{(i)}$ are then given by

$p_{\mathrm{t}}^{(i)}=\sigma_{0} \frac{j \omega c_{\mathrm{T}}}{k_{\mathrm{r}} \alpha_{\mathrm{f}}} \frac{\rho_{\mathrm{f}} Z \alpha_{\mathrm{eff}}}{R} \frac{H_{1}(\zeta R)}{H_{0}(\zeta R)}$,

$p_{\mathrm{inc}}^{(i)}=\sigma_{0} \frac{\rho_{\mathrm{f}} c_{\mathrm{T}}^{2}}{\rho V_{S}^{2}}\left(\frac{1-2 V_{S}^{2} / V_{P}^{2}}{1-c_{\mathrm{T}}^{2} / V_{P}^{2}}\right)$,

where $\sigma_{0}$ is the amplitude of the normally incident plane $\mathrm{P}$ wave, $j=\sqrt{-1}$ the imaginary unit; $\omega$ the angular frequency; $k_{\mathrm{r}}$ the radial wave number for a rigid, nondeformable fracture (a function of $L_{0}$ ); $\alpha_{\mathrm{f}}$ the fluid velocity; $\alpha_{\text {eff }}$ the effective fluid velocity in the fracture (a function of $L_{0}$ and $Z$ ); and $R$ the borehole radius. $H_{n}$ denotes the Hankel function of the first kind of order $n, \zeta$ the effective radial wave number (a function of $L_{0}$ and $Z$ ), and $\rho$ the density of the embedding background rock. $V_{P}$ and $V_{S}$ are the $\mathrm{P}$-wave and $\mathrm{S}$-wave velocity in the background rock, respectively. Note that $\sigma_{0}$ drops out of Eq. (4) due to the ratio of $p_{\mathrm{t}}^{(i)}$ and $p_{\text {inc }}^{(i)}$.

When a tube wave propagating along the borehole interface encounters a fracture, fluid flow from the borehole into the fracture is triggered. This leads to reflection and transmission of tube waves. This process is described with the scattering potential $\phi_{s}$ in the frequency domain:

$\phi_{S}(z)=j \omega \sum_{i=1}^{N} \eta^{(i)} \delta\left(z-z_{i}\right)$,

where $\eta$ is the interface compliance given by

$\eta=-\frac{2 \zeta}{R} \frac{L_{0}}{k_{\mathrm{r}}^{2} \alpha_{\mathrm{f}}^{2} \rho_{\mathrm{f}}} \frac{H_{1}(\zeta R)}{H_{0}(\zeta R)}$.
Note that the interface compliance differs from the fracture compliance. It linearly relates the velocity discontinuity $\Delta V$ across the fracture to the acoustic pressure $p: \Delta V=j \omega \eta p$ (Minato and Ghose, 2017). Note also that, in our implementation of this forward solver, tube waves that are generated at borehole enlargements, such as washouts and bitsize changes, or at high acoustic impedance contrasts due to lithological changes are not taken into account. Further details about the tube-wave generation and scattering potentials, and the algorithm itself, can be found in Minato and Ghose (2017).

For the forward operator $G$ as described so far, we assumed the fractures to be horizontally oriented. To account for arbitrary incidence angles, we have extended the above algorithm for the forward operator $G$, following the description given by Minato et al. (2017).

To improve the estimation of the fracture compliance $Z$, we have extended the forward operator of Minato and Ghose (2017) to include transmission losses of $P$ waves across fractures, by using the angle-dependent transmission coefficient described by the linear slip theory (Schoenberg, 1980). Accordingly, the $\mathrm{P}$ - and $\mathrm{S}$-wave reflection coefficients $R_{P}$ and $R_{S}$, as well as the $\mathrm{P}$ - and $\mathrm{S}$-wave transmission coefficients $T_{P}$ and $T_{S}$, for an incoming $\mathrm{P}$ wave are given by

$$
\begin{aligned}
& {\left[\begin{array}{llll}
-p_{1} & \gamma_{1} \cos \left(\psi_{1}\right) & p_{2} & \gamma_{2} \cos \left(\psi_{2}\right) \\
\gamma_{-1 \cos \left(\theta_{1}\right)} & q_{1} & -\cos \left(\theta_{2}\right) & \gamma_{2} \cos \left(\theta_{2}\right) \\
\cos \left(\theta_{1}\right) & -\sin \left(\psi_{1}\right) & \sin \left(\theta_{2}\right)-j \omega Z_{2} \gamma_{2} \cos \left(\theta_{2}\right) & -q_{2}\left(\cos \left(\psi_{2}\right)+j \omega Z_{2} q_{2}\right. \\
\cos \left(\theta_{1}\right) & -\sin \left(\psi_{1}\right) & \cos \left(\theta_{2}\right)-j \omega Z_{N} p_{2} & \sin \left(\psi_{2}\right)-j \omega Z_{N} \gamma_{2} \cos \left(\psi_{2}\right)
\end{array}\right]} \\
& {\left[\begin{array}{l}
R_{P} \\
R_{S} \\
T_{P} \\
T_{S}
\end{array}\right]=\left[\begin{array}{l}
p_{1} \\
\gamma_{1} \cos \left(\theta_{1}\right) \\
\sin \left(\theta_{1}\right) \\
\cos \left(\theta_{1}\right)
\end{array}\right],}
\end{aligned}
$$

where

$$
\begin{aligned}
& \gamma_{m}=2 \rho_{m} V_{S_{m}} \sin \left(\psi_{m}\right), \\
& p_{m}=\rho_{m} V_{P_{m}}-\gamma_{m} \sin \left(\theta_{m}\right), \\
& q_{m}=\rho_{m} V_{S_{m}} \cos ^{2}\left(\psi_{m}\right)-\frac{1}{2} \gamma_{m} \sin \left(\psi_{m}\right),
\end{aligned}
$$

with the superscript $m$ being 1 for the medium above and 2 for the medium below the fracture. The angles $\theta_{m}$ and $\psi_{m}$ refer to the $\mathrm{P}$-wave and the $\mathrm{S}$-wave reflection angles if the superscript $m$ is 1 and to the corresponding transmission angles if the superscript $m$ is $2 . Z_{T}, Z_{N}$, and $\rho$ denote the fracture compliance in the transverse direction (parallel to the fracture), the fracture compliance in the normal direction (perpendicular to the fracture), and the density, respectively. Note that in this study we assume for simplicity that $Z=Z_{T}=Z_{N}$. We solve Eq. (10) for the four coefficients, but we only use the transmission coefficient $T_{P}$ to reduce the amplitude of the $\mathrm{P}$ wave after having crossed a fracture, because we do not consider reflections or $\mathrm{S}$ waves in this study.

In order to fit the observed data, we implemented the forward operator of Minato and Ghose (2017) such that 
the following features are explicitly included: (1) geometrical spreading of $\mathrm{P}$ waves is accounted for by multiplying Eq. (7) with $1 / z$. Note that other attenuation mechanisms of the $\mathrm{P}$ wave, besides geometrical spreading and transmission losses across fractures, are neglected. (2) The algorithm assumes a uniform embedding background medium. To account for P-wave velocity changes above the considered borehole section, we introduce a variable source depth. This is an auxiliary parameter estimated during the inversion. (3) The algorithm assumes an isotropic background medium. As the particle motion of a $\mathrm{P}$ wave is different from that of a tube wave in the elastic medium surrounding the borehole, the two wave types are sensitive to the background medium properties in different directions. Therefore, taking anisotropy into account is important for fitting observed data. We do this by estimating different effective isotropic shear moduli for the $\mathrm{P}$ wave and for the tube wave. Thus, the shear modulus $\mu$ in Eq. (5) becomes $\mu_{t}$, the tube-wave shear modulus.

Due to the nonlinearity of the problem, we cannot infer the posterior PDF directly; instead we need to infer it by sampling the prior PDF and the likelihood according to Relation (1). For this, we chose to use a Markov chain Monte Carlo (MCMC) approach. This algorithm walks randomly through the solution space, accepting or rejecting proposed models $\mathbf{m}_{\text {prop }}$, which are drawn from a symmetric proposal distribution, with the Metropolis acceptance probability $\alpha$ (Metropolis et al., 1953):

$\alpha=\min \left\{1, \frac{L\left(\mathbf{m}_{\text {prop }} \mid \mathbf{d}\right) p\left(\mathbf{m}_{\text {prop }}\right)}{L\left(\mathbf{m}_{\text {cur }} \mid \mathbf{d}\right) p\left(\mathbf{m}_{\text {cur }}\right)}\right\}$,

where $\mathbf{m}_{\text {cur }}$ is the model at the current location of the Markov chain. We use the $\operatorname{DREAM}_{(\mathrm{ZS})}$ algorithm (ter Braak and Vrugt, 2008; Laloy and Vrugt, 2012) to accomplish the sampling of Relation (1) efficiently. DREAM $(\mathrm{ZS})$ allows for a fast convergence towards the posterior PDF due to parallel and interacting Markov chains as well as a model-proposal scheme that uses a database of previously accepted models to avoid sampling areas of low posterior probability and focusing on the interesting areas of the solution space.

The viability and accuracy of the algorithm have been tested and verified in a variety of synthetic case studies, an example of which is shown in the next section. Subsequently, we apply the proposed inversion scheme to hydrophone VSP data acquired at the underground Grimsel Test Site in the central Swiss Alps.

\section{Results: a synthetic example with real noise}

Before applying our inversion algorithm to observed data, we ran tests on synthetic data to ensure that the algorithm performs as expected. As in these experiments the same forward solver was used for the generation and the inversion of the data, the corresponding results only allow conclusions to be drawn with regard to the inversion algorithm itself, but not with regard to the information content of the data. The test case shown here features two fractures at 10 and $19 \mathrm{~m}$ depth. The receiver spacing is $1 \mathrm{~m}$. To make this synthetic study more pertinent and challenging, we contaminated the dataset with actual ambient noise from a corresponding field dataset at the underground Grimsel Test Site in Switzerland. The resulting data are plotted in Fig. 1a.

This synthetic test differs from the field-data example shown in the next section in two ways: (1) it uses as a forward solver the algorithm proposed by Minato and Ghose (2017) and Minato et al. (2017) without taking transmission losses, geometrical spreading for $\mathrm{P}$ waves, velocity changes above the considered borehole section, or anisotropy into account, because these features are not present in the underlying synthetic data. (2) While the wavelet is based on a mean trace for the field data, we treat it as unknown and, thus, estimate it in the synthetic example. We do this by inferring the coordinates of six pilot points, from which we obtain the wavelet by a shape-preserving piecewise cubic interpolation (Hunziker et al., 2019).

The inversion was run once with three parallel Markov chains. Figure 2 shows the estimate of the hydraulic fracture aperture and the mechanical compliance for the two fractures as a function of the number of forward simulation steps. For all four unknowns, the three chains converge nicely to the true values. This behavior illustrates that the algorithm works properly even when the data are contaminated with correlated, realistic noise.

Simulated data based on a model proposed at the end of the first Markov chain agree very well with the input data (Fig. 1a, b). Note that, besides the direct $\mathrm{P}$ wave (1) and the tube waves generated at fractures (2), the tube waves reflected at fractures (3) are also visible. The latter are visible neither in the noise-contaminated input data nor in the actual field data.

\section{Results: a real-data example}

The VSP data, considered in the following, were recorded in crystalline rocks at the underground Grimsel Test Site in Switzerland using a 12-receiver hydrophone string with a receiver spacing of $1 \mathrm{~m}$. In the course of the experiment, the hydrophone string was repositioned, such that, the recorded traces are separated by $0.5 \mathrm{~m}$. The borehole had a diameter of $0.147 \mathrm{~m}$. As a source, a single-handed $2 \mathrm{~kg}$ hammer was used at the wellhead, which excited frequencies between 0.1 and $4 \mathrm{kHz}$ with a peak around $1.5 \mathrm{kHz}$. In this study, we consider a $20 \mathrm{~m}$ long subsection between 17 and $37 \mathrm{~m}$ depth, consisting of 41 hydrophone receiver positions. Through visual inspection of the VSP dataset, complemented by evidence from optical and acoustic televiewer data (Krietsch et al., 2018), three fractures at 23.5, 23.9, and $25 \mathrm{~m}$ depth have been identified. 
(a) Test data

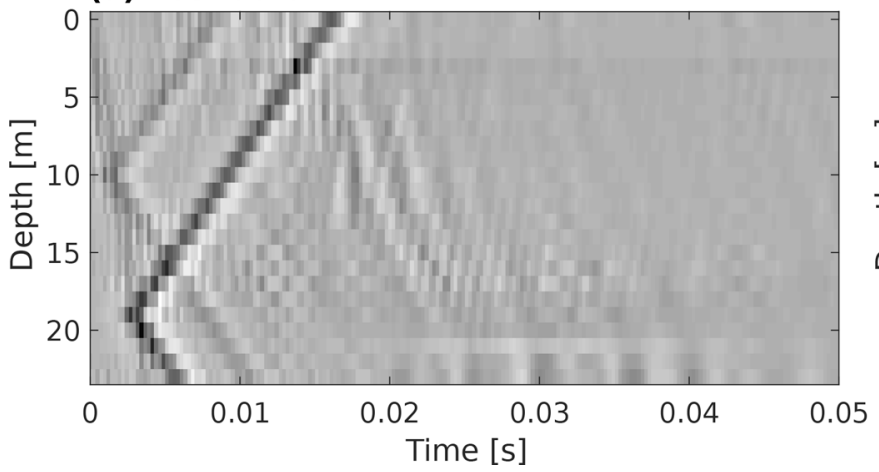

(b) Modeled data based on inversion results

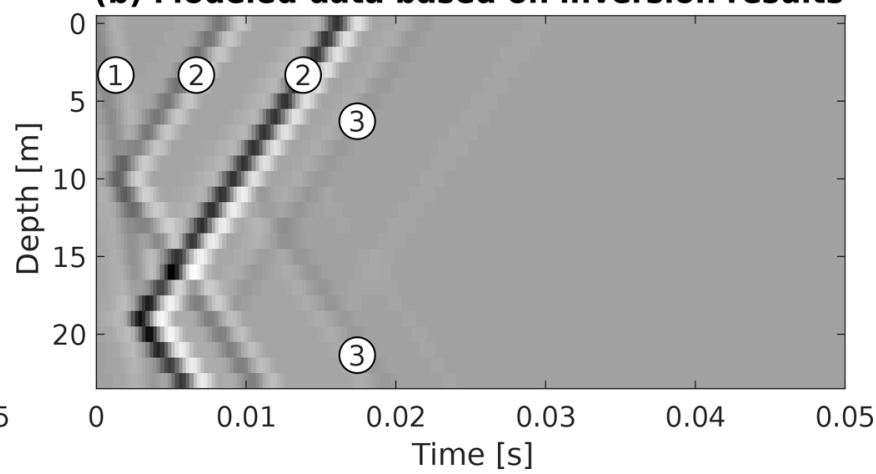

Figure 1. (a) Synthetic test data featuring two fractures at 10 and $19 \mathrm{~m}$ depth contaminated with ambient noise from observed hydrophone VSP data acquired at the underground Grimsel Test Site in Switzerland; (b) simulated data based on an inferred model at the end of a Markov chain. (1) denotes the direct $\mathrm{P}$ wave, (2) the tube waves generated at the fractures, and (3) the tube waves reflected at the fractures.

(a)
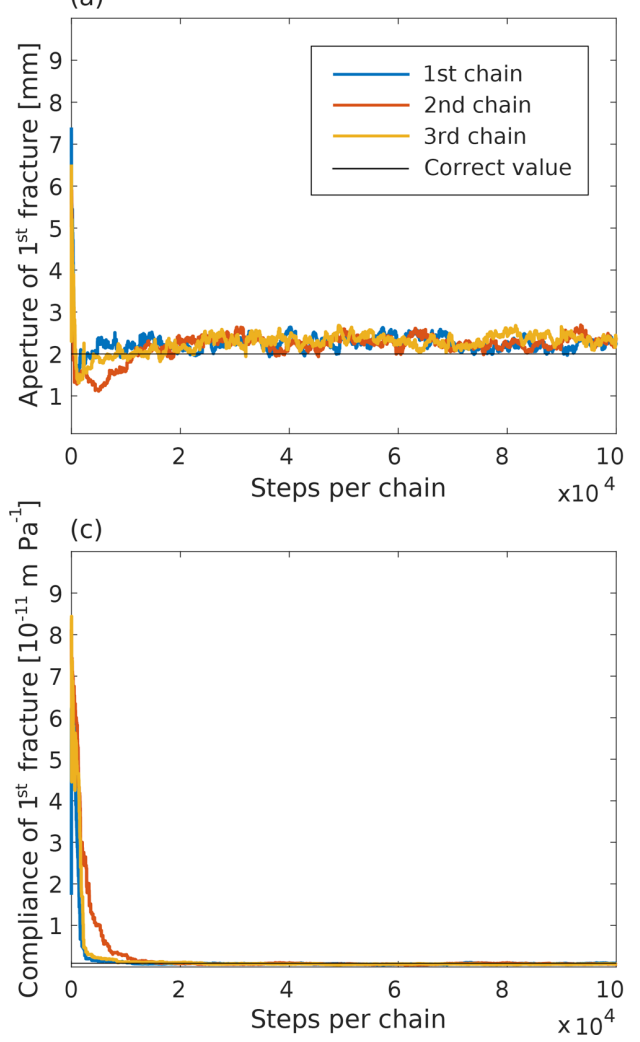

(b)
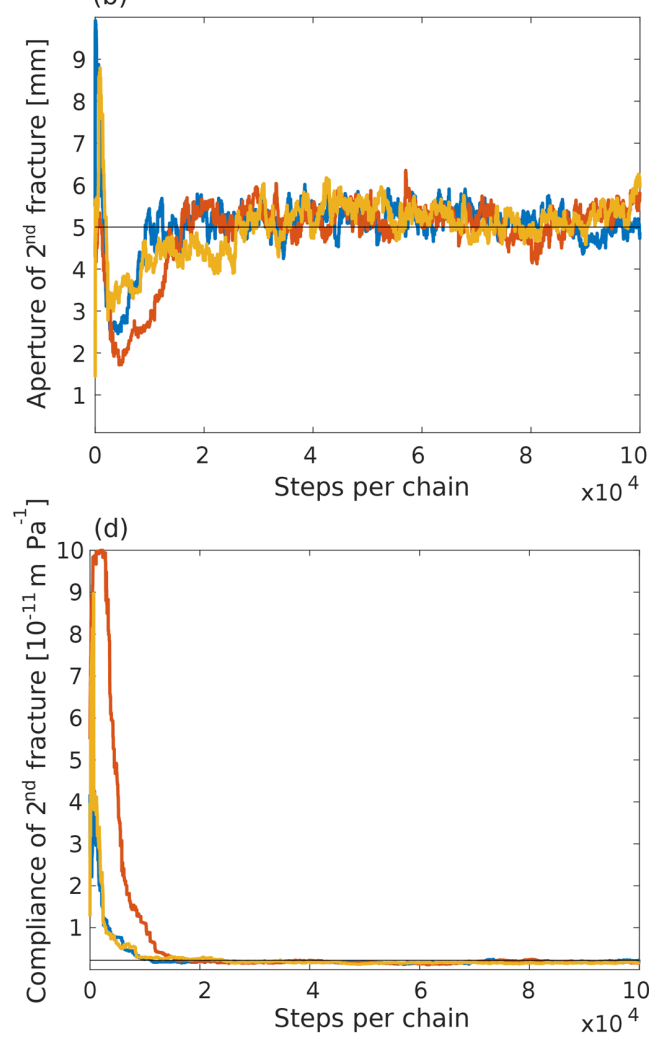

Figure 2. Estimates of (a-b) the aperture and (c-d) the compliance of the two fractures as functions of the number of forward modeling steps. The horizontal black lines denote the corresponding values used to generate the synthetic data shown in Fig. 1a.

Preprocessing of the data included a gentle bandpass filter to suppress high-frequency noise, a static shift correction to remove positioning errors, and a cosine taper to blank out the later-arriving $\mathrm{S}$ wave and associated tube waves. The data after preprocessing are shown in Fig. 3. The $\mathrm{P}$ wave and the tube waves are clearly visible. However, scattered tube waves, as described by Eq. (8), are weak in amplitude and drop below the noise level. As the first and the second fracture are located closely together, the corresponding tube waves overlap, which poses a particular challenge for the inversion process. Before the data are supplied to the inversion algorithm, we separate the $\mathrm{P}$ wave from the tube waves, apply a move-out correction to the $\mathrm{P}$ wave, and then calculate a mean trace. A time-gated version of this mean trace with a length of $10 \mathrm{~ms}$ then serves as the estimate of the source wavelet. 


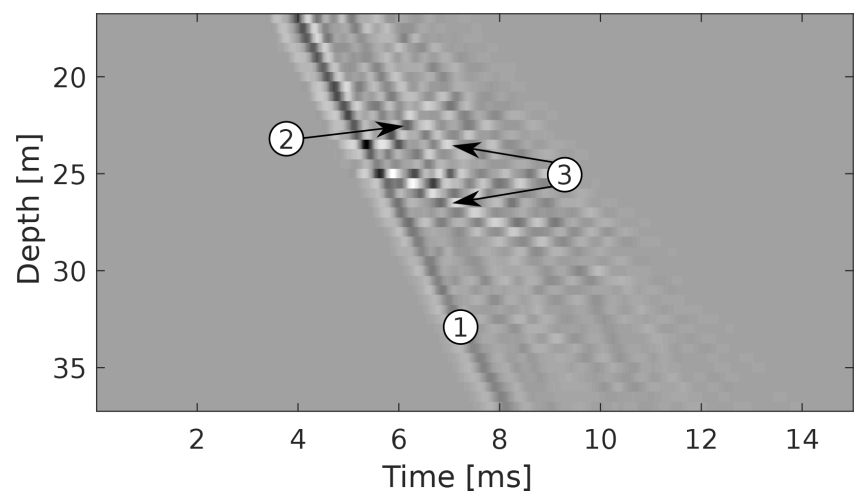

Figure 3. Observed hydrophone VSP data considered in this study. (1) denotes the downgoing $\mathrm{P}$ wave, (2) the upgoing tube wave due to the fractures at 23.5 and $23.9 \mathrm{~m}$ depth, and (3) the up- and downgoing tube wave due to the fracture at $25 \mathrm{~m}$ depth. Note the amplitude decay associated with the $\mathrm{P}$ wave.

For this problem with three fractures, we have 15 unknowns, which are specified in Table 1. Three unknowns are related to the background rock. These are the bulk and shear moduli of the formation and a separate shear modulus used for the tube waves. As outlined above, we use separate shear moduli for the $\mathrm{P}$ wave and for the tube waves as a first-order approximation to account for anisotropy, which was estimated to be approximately $10 \%$ at the considered site (Wenning et al., 2018). The next nine unknowns are related to the fractures. For each of the three fractures, we estimate the hydraulic aperture, the compliance, and the depth. The forward solver also takes the fracture inclination into account. However, as tests on synthetic data showed that the fracture inclination cannot be inferred with high confidence, we assume that the inclination is known from televiewer data. The remaining three unknowns are algorithmic "tuning" parameters without any physical meaning. The first parameter of this group is the source depth. While the actual source location is known, we estimate the source depth for a fictitious homogeneous background medium to accommodate possible variations of the background medium parameters above the section under consideration. If the background rock is indeed homogeneous, the estimated source depth will correspond to the true source depth. The other two tuning parameters are used to emulate attenuation of the tube waves. As tube waves propagate along the borehole, they do not suffer from geometrical spreading as, for example, the $\mathrm{P}$ wave does (Fig. 3). However, tube waves are attenuated due to inelastic effects or scattering. To account for this, we dampen the tube waves using an exponential function defined by a shift factor, which specifies when the damping starts, and an exponent, which specifies the damping rate.

We ran our algorithm three times to ensure that it successfully locates the posterior PDF and does not get stuck in a local minimum. Each time, three parallel Markov chains were used to explore the parameter space. More chains would have

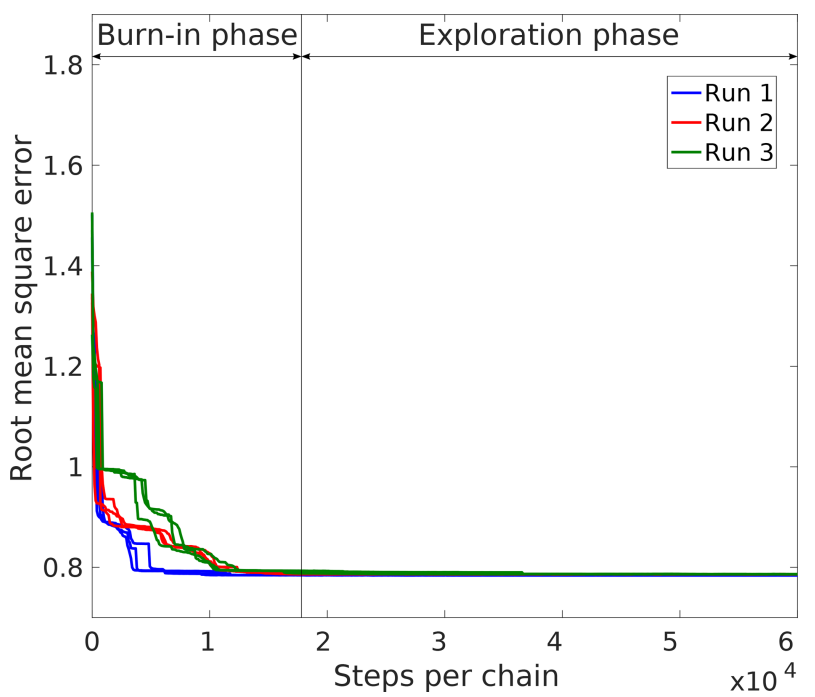

Figure 4. RMSE weighted by the standard deviation of the data error for the three inversion runs of the observed VSP data shown in Fig. 3. As the estimate of the standard deviation of the data error is fixed at a high value, the RMSE drops below 1 . The vertical black line indicates the separation of the burn-in and the exploration phases, associated with the MCMC search of the parameter space.

allowed for a more comprehensive exploration of the solution space, but would also have required more computational resources. Three chains are in our experience sufficient to exhaustively explore a 15-dimensional solution space well, such that the posterior PDF is found in most of the runs. The development of the root mean square error (RMSE) is plotted in Fig. 4 for each Markov chain. Here, we weight the RMSE with the standard deviation of the data error. This means that, ideally, the weighted RMSE should converge to a value of 1 , with smaller values indicating that the data are over-fitted and larger values implying that not all the data can be explained by the proposed model. With the objective to force the algorithm to more extensively explore the posterior distribution, we fix the standard deviation of the data error at a relatively high value, which is larger than corresponding estimates obtained in previous inversion runs. Figure 4 shows that all runs converge to a stable RMSE value, which, as the data error is fixed at a high value, is smaller than 1 . Before reaching a stable RMSE, the algorithm explores the complete solution space in search of the posterior PDF. This is referred to as the burn-in phase. Subsequently, the algorithm is expected to have located the posterior PDF and to explore it in the course of the remaining iterations.

In order to assess whether the Markov chains have converged sufficiently to allow for a reliable estimation of the posterior PDF, we calculate the so-called potential scale reduction factor $\hat{R}$ (Gelman and Rubin, 1992). Considering only the part of the Markov chains after burn-in, $\hat{R}$ compares the variance of the individual Markov chains with the overall variance of all the chains together. Usually, convergence 
Table 1. Unknowns of the inverse problem and their prior ranges subdivided by horizontal lines into three groups. The first group from the top comprises the background medium parameters, the second group the fracture parameters, and the third group algorithmic "tuning" parameters.

\begin{tabular}{llrc}
\hline & Unknown & Prior range & Unit \\
\hline Background properties & bulk modulus of the background rock & $20-80$ & $\mathrm{GPa}$ \\
& $\begin{array}{l}\text { shear modulus of the background rock for the P wave } \\
\text { shear modulus of the background rock for the tube wave }\end{array}$ & $15-33$ & $\mathrm{GPa}$ \\
& $2-50$ & $\mathrm{GPa}$ \\
\hline \multirow{2}{*}{ Fracture properties } & aperture of first fracture & $10^{-4}-10^{-2}$ & $\mathrm{~m}$ \\
& aperture of second fracture & $10^{-4}-10^{-2}$ & $\mathrm{~m}$ \\
& aperture of third fracture & $10^{-4}-10^{-1}$ & $\mathrm{~m}$ \\
& compliance of first fracture & $10^{-15}-10^{-10}$ & $\mathrm{mPa} \mathrm{P}^{-1}$ \\
& compliance of second fracture & $10^{-15}-10^{-10}$ & $\mathrm{~m} \mathrm{~Pa}-1$ \\
& compliance of third fracture & $10^{-15}-10^{-10}$ & $\mathrm{~m} \mathrm{~Pa}$ \\
& depth of first fracture & $23.0-24.0$ & $\mathrm{~m}$ \\
& depth of second fracture & $23.4-24.4$ & $\mathrm{~m}$ \\
& depth of third fracture & $24.5-25.5$ & $\mathrm{~m}$ \\
\hline \multirow{2}{*}{ "Tuning" parameters } & source depth & $1.5-1.6$ & $\mathrm{~m}$ \\
& tube-wave attenuation shift factor & $0.001-0.02$ & $\mathrm{~s}$ \\
& tube-wave attenuation exponent & $0.0-1000.0$ & - \\
\hline
\end{tabular}

is considered to be reached if $\hat{R}$ is smaller than 1.2 for all parameters. In this example, considering a burn-in phase of $30 \%$ of the complete chains, we get $\hat{R}<2$ for most parameters, but only approximately a third of the parameters reach $\hat{R}<1$.2. Consequently, the posterior PDF has not been fully explored. Therefore, we do not plot posterior PDFs for the inferred parameters. Instead, we show the development of the Markov chains as a function of iteration number. Although proper convergence has not been achieved, the inferred models explain the data well. However, other models, not sampled by the Markov chains, might explain the data equally well. Hence, longer chains would be necessary to ensure a comprehensive exploration of the posterior PDF.

The acceptance rate specifies how many of the tested models are accepted. A too-high acceptance rate generally implies that only models in the immediate neighborhood of the current model are explored, while a too-low acceptance rate means that computational resources are wasted by testing unrealistic models. Ideally, the acceptance rate ranges between 10 and $30 \%$. In our case, it lies between 10 and $20 \%$ for runs one and two and around $5 \%$ for run three.

The most interesting inferred parameters are the apertures and compliances of the fractures, and to a lesser extent the background rock properties. The development of these unknowns as a function of the number of iterations is plotted for all three runs in Fig. 5. For the aperture of the first fracture (Fig. 5a), the algorithm either finds a very large value of $10 \mathrm{~mm}$ (run one) or a rather small one of less than $0.5 \mathrm{~mm}$ (runs two and three). Interestingly, the opposite is the case for the second fracture (Fig. 5b). Here, run one suggests a small fracture aperture, and runs two and three a large one. As mentioned earlier, the first two fractures are very close together, at 23.5 and $23.9 \mathrm{~m}$ depth, respectively. Hence, the corresponding tube waves overlap. The algorithm, thus, finds that one fracture must have a much larger aperture than the other, but it cannot determine which one is which. This leads to a bimodal posterior PDF featuring two equally probable modes. The estimated compliance values for these two fractures behave similarly (Figs. $5 \mathrm{~d}$ and e), although the difference between the runs is smaller.

The vertical axis of the plots in Fig. 5 represents the prior range. In the cases where the first or the second fracture is found to have a large aperture, the inferred value is actually located at the upper limit of the prior range. This means that the algorithm would propose even larger values if it were allowed to do so. We have not extended the prior range, because (1) even larger fracture apertures seem unrealistic and (2) the models found within this prior range are able to explain the data well.

The posterior PDF for the estimates of the aperture of the third fracture is unimodal (Fig. 5c). At the location of the third fracture, televiewer data (Krietsch et al., 2018) also indicate the presence of a larger shear zone. As we were not sure if the observed tube wave stems from the shear zone or the fracture, we extended the prior range of the aperture for this fracture by 1 order of magnitude to be able to accommodate the complete shear zone. However, all three runs suggest a small aperture of less than $1 \mathrm{~mm}$, which clearly indicates that the tube wave is generated by the fracture and not by the shear zone.

For the bulk and shear modulus of the background (Figs. $5 \mathrm{~g}$ and $\mathrm{h}$ ), we observe a similar behavior to that for the fracture apertures of the first and the second fracture: if the bulk modulus is large, then the shear modulus is small 

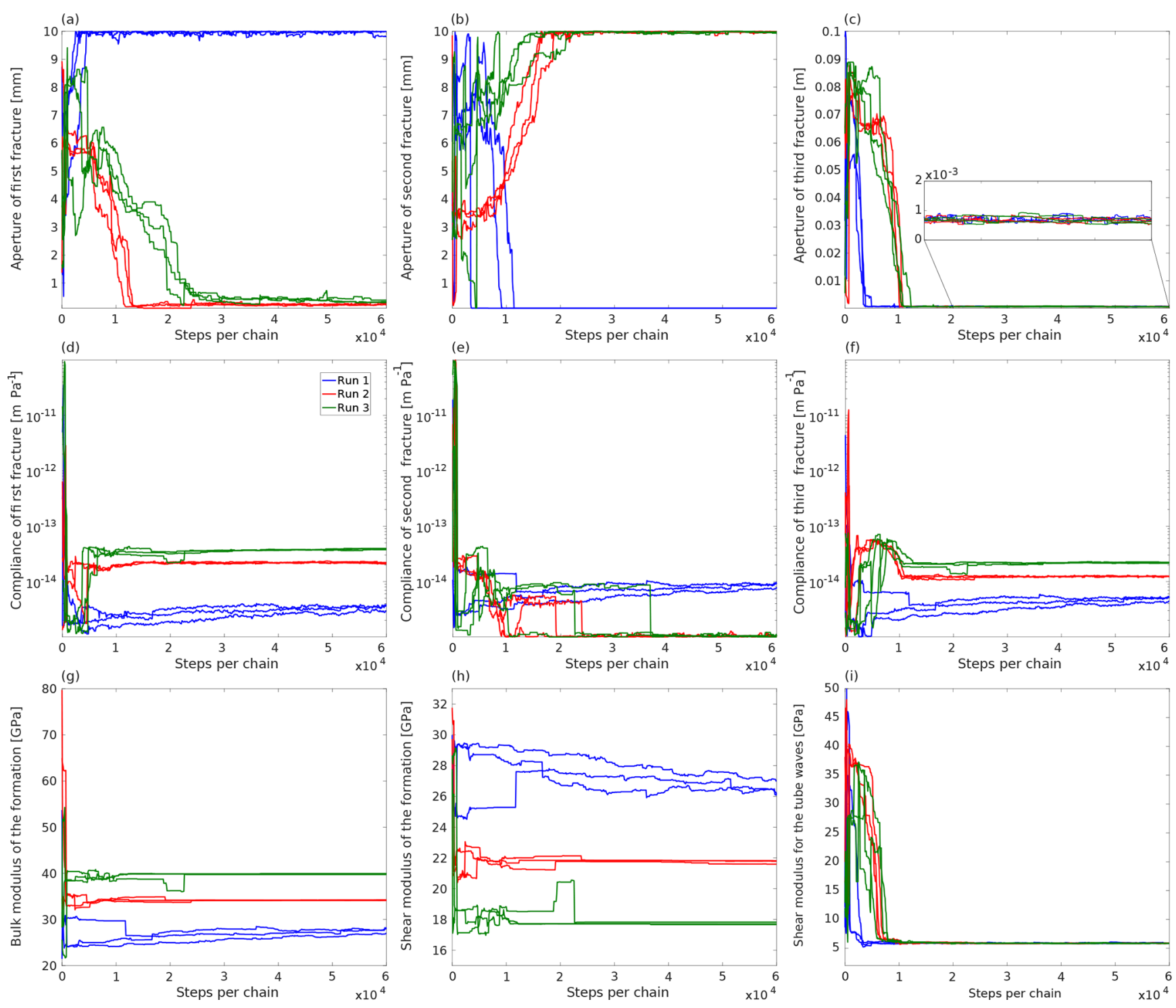

Figure 5. Development of the most relevant unknowns for the three MCMC inversion runs of the observed VSP data shown in Fig. 3: (a-c) apertures of the three fractures, $(\mathbf{d}-\mathbf{f})$ corresponding compliances, and (g-i) elastic moduli.

and vice versa. Both parameters are constrained by two observables: (1) the P-wave velocity by the move-out of the $\mathrm{P}$ wave and (2) the transmission coefficient by the amplitude loss of the $\mathrm{P}$ wave across fractures. However, these two observables are insufficient to constrain the background moduli adequately, thus leaving some degree of ambivalence in the final estimates. Conversely, the shear modulus used for the calculation of the tube-wave velocity is well constrained (Fig. 5i), because there is no trade-off with other parameters.

As the RMSE in Fig. 4 is the same for all runs, the two modes of the posterior PDF identified by the algorithm explain the data equally well. To further illustrate this, we compare in Fig. 6 synthetic data based on the inversion results presented in Fig. 5 with the observed data. We generate the synthetic data using the last model of the third Markov chain of run one (blue in Fig. 6a), in which the first fracture is inferred as having a large aperture, and of run two (red in Fig. 6b), in which the second fracture has a large aperture. The observed data are plotted in black. Although we use a semi-analytic forward solver - which is inherently subject to a number of rather stringent assumptions, such as a homogeneous background medium - both synthetic datasets fit the observed data remarkably well.

\section{Discussion}

Based on the interpretation of the optical televiewer data by Krietsch et al. (2018), the three fractures considered in this study have apertures of $6.4,1.7$, and $0.0 \mathrm{~mm}$. These are the 

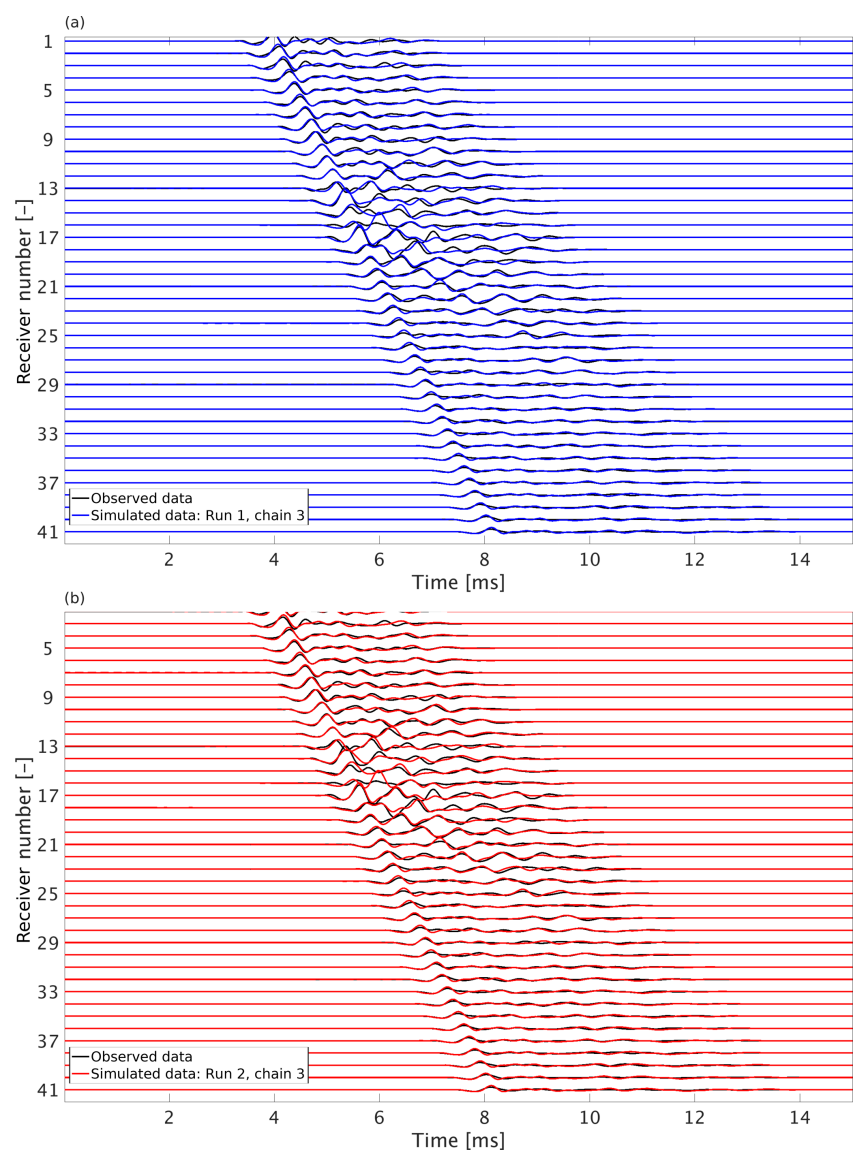

Figure 6. Comparison between simulated (colored) and observed (black) data: (a) run one and (b) run two.

fracture apertures at the borehole wall, which are not identical to the hydraulic fracture apertures inferred in this study. While the former represents the actual aperture at the interface between the borehole and the fracture, the latter is an average of the hydraulic aperture over the rock volume in the vicinity of the borehole sampled by the VSP data. In spite of these differences, the televiewer data can, for example, help identify the correct mode in the case of multimodal posterior distributions. In our case, the televiewer data indicate that the first fracture has a larger aperture than the second one, confirming that the modal aperture distribution identified by run one is realistic. However, run one infers for the second fracture a much smaller aperture than indicated by the televiewer data. This indicates that, although the fracture has according to the televiewer an aperture in excess of $1 \mathrm{~mm}$ at the borehole wall, it is likely to be much thinner away from the borehole. The aperture of the third fracture is smaller than the vertical resolution of the optical televiewer of $0.21 \mathrm{~mm}$. Similarly, we also obtain a very small fracture aperture, which is estimated by our algorithm to be below $1 \mathrm{~mm}$. Concerning the fracture compliances, we can compare our results with those of Barbosa et al. (2019), who present corresponding estimates for the same borehole section based on full-waveform sonic log data. They estimated fracture compliances which are approximately 1 order of magnitude higher than our results $\left(9.9 \times 10^{-13} \mathrm{~m} \mathrm{~Pa}^{-1}\right)$. Potential reasons for this difference might be that the full-waveform sonic data were measured at significantly higher frequencies $(\sim 20 \mathrm{kHz})$ than our VSP data and that the fracture compliances tend to be frequency dependent (e.g., Pyrak-Nolte, 1992; Nakagawa, 2013). Another difference between the two studies is the incidence angle. While Barbosa et al. (2019) assume normal incidence of the $\mathrm{P}$ wave on the fractures, this study accounts for the dip angle of the fractures derived from televiewer data, which ranges from 62 to $77^{\circ}$ with regard to the horizontal.

A bit puzzling is the remarkably low estimate of the tubewave shear modulus of only about $6 \mathrm{GPa}$ (Fig. 5i). This parameter is very well constrained, as it is the only free parameter in Eq. (5), which may, however, be too simplistic for the following three reasons: (1) Eq. 5 is derived in the low-frequency regime, and its validity for higher frequencies is limited. (2) Attenuation of tube waves, as for example through scattering on the borehole tool or inside the damaged zone surrounding the borehole, was not accounted for when estimating the tube-wave shear modulus. (3) Anisotropy is not taken into account completely. Thus, while the resulting tube-wave velocity is correct, as can be seen by the excellent fit between the observed and synthetic data, the corresponding shear modulus appears to be underestimated in order to correct for physical effects neglected in Eq. (5). Incorporating attenuation into the tube-wave velocity equation can be done by implementing Eqs. (5)-(17) of White (1983) including the impedance of the borehole wall, and accommodating anisotropy can be done by one of the methods presented by Karpfinger et al. (2012). This, however, is beyond the scope of the present study.

From an inversion perspective, the most interesting point of these results is that two modes of the posterior PDF were identified. This showed that having the first fracture with a large aperture, while the second fracture is thin, is similarly probable to the opposite scenario. Note that a deterministic approach would have provided only one result without any indication that there is another mode that can explain the data equally well, whereas our Bayesian approach clearly supplied us with both options. This nicely demonstrates the value of stochastic inversion approaches.

A downside of our Bayesian approach is its enormous computational cost. Most of it is spent in the forward steps to simulate VSP data for the proposed model. We have optimized the forward simulation by parallelizing over frequencies. Still, one inversion run with three parallel Markov chains and 60000 forward steps per chain took approximately $14 \mathrm{~d}$ to complete using one node (48 AMD Opteron 6174 processors at $2.2 \mathrm{GHz}$ ) of our cluster. However, the inversion would run 3 times faster if each of the three Markov chains were run on a different node. We did not do this due to limited availability of resources. In any case, we argue that 
the computation time is well spent, since the results obtained are much more comprehensive than those that would be obtained through a deterministic inversion, as they allow, as explained above, multiple modes of the posterior PDF to be discovered. Furthermore, stochastic inversion approaches do not really depend on the starting model. This is in stark contrast to deterministic full-waveform inversion approaches, which require starting models whose forward response deviates from the forward response of the true model by less than half a wavelength (Virieux and Operto, 2009).

For the real-data example, we have decided not to estimate the source wavelet during the inversion process, although the corresponding algorithm was developed and successfully applied for synthetic test cases as demonstrated in the first results section. The reason is that the source wavelet of the observed data includes extensive reverberations and is, thus, extremely long and complicated. Estimating it as part of the inversion procedure would have required more than doubling the amount of unknowns, which would have rendered the problem unnecessarily complex.

An important limitation of our forward model, and indeed of virtually all fracture-based tube wave models, is that fracture aperture and compliance are correlated. This means that the inversion algorithm tends to compensate for an overestimation of the fracture aperture by underestimating the fracture compliance. Therefore, we observe that a large fracture aperture for the first fracture is accompanied by a relatively small fracture compliance (Figs. 5a and d). This is supposed to be mitigated in our approach, because the estimate of the fracture compliance is constrained not only by the tube-wave amplitude but also by the reduction of the P-wave amplitude when a fracture is crossed (Schoenberg, 1980). However, the transmission coefficients calculated for the estimated parameters are very close to 1 , and hence the effect of this constraint is relatively weak. As the Markov chains are not oscillating all over the prior range, and as the obtained values are reasonable, we can conclude that this compensation is rather limited.

Inspecting the difference between the observed and the forward modeled data shows that the largest discrepancies are found at the fracture locations. This indicates that the transmission loss of the $\mathrm{P}$ wave across fractures may not be reproduced properly in the synthetic data. However, as this affects only the $P$ wave around the fracture locations, the impact on the RMSE is limited. A possible way to improve this issue might be to define a weighting function that peaks at the fracture locations to force the algorithm to obtain a better data fit at these locations, and thus, find a more accurate transmission coefficient. The downside of this, however, is that the weights are new tuning parameters that need to be adjusted through a time-consuming process, which was not feasible to accomplish in the scope of this study.

Limitations of our implementation of the forward operator are its inability to account for scatterers, impedance contrasts related to lithological changes, and borehole enlargements.
If corresponding effects are present in the data, they might need to be filtered out prior to inversion. Similarly, changes in the P-wave velocity are not taken into account. If these are present, the data need to be cut into smaller pieces with constant $\mathrm{P}$-wave velocity. Changes in $\mathrm{P}$-wave velocity above the considered borehole section are taken into account by virtually shifting the source depth. The algorithm is also not able to take $\mathrm{S}$ waves and corresponding tube waves into account. In our dataset, events of this kind were indeed present and needed to be muted before applying our inversion algorithm to the dataset.

\section{Conclusions}

We have developed a Bayesian MCMC full-waveform inversion algorithm based on a semi-analytical forward solver to simultaneously infer the aperture and compliance of individual fractures from corresponding tube-wave data. We mitigate the correlation between fracture aperture and compliance by constraining the fracture compliance by two independent observables: (1) the tube-wave amplitude relative to the P-wave amplitude and (2) the amplitude loss of the $\mathrm{P}$ wave across a fracture. The algorithm was applied to a field dataset acquired in crystalline rock at the underground Grimsel Test Site in Switzerland. The subsection of the VSP dataset considered contained three fractures, of which two are very close together. The algorithm identified two equally probable modes in the posterior PDF: either the first fracture features a large aperture and the second fracture a small one or vice versa. In other words, from the information provided, the algorithm can determine that one fracture is larger than the other, but it cannot determine which one is thick and which one is thin. The identification of these two modes clearly illustrates a major advantage of stochastic inversion algorithms as compared to their deterministic counterparts. The latter would not have identified these two modes and would have provided just one of the two possible solutions. Our case study also shows that in a complex geological environment with multiple, closely spaced fractures the hydraulic apertures of individual fractures cannot be determined. However, the method can still provide an effective fracture aperture distribution of a package of fractures. The inferred apertures in our example are consistent with televiewer data, and the inferred compliances are roughly in the same range as those derived from sonic logs at the same site. The data fit is remarkably good, especially when considering the semianalytical nature of the forward solver and the inherent assumptions it relies on, as well as the rather complex character of the observed hydrophone VSP data.

Code availability. The forward solver can be downloaded from https://github.com/rockphysicsUNIL/tube_wave_forward_solver (Hunziker et al., 2020). 
Author contributions. JH developed the inversion, contributed to the data analysis and wrote the majority of the manuscript. AG collected and processed the hydrophone VSP data, and contributed to the scientific discussion. SM contributed to the analysis of the results and the final manuscript. NDB contributed to the development of the forward solver and the analysis of the data and the corresponding scientific discussion. EC contributed to the development of the forward solver, the scientific discussion and the understanding of the dataset. KH acted as project leader and participated in the research effort and the manuscript preparation.

Competing interests. The authors declare that they have no conflict of interest.

Acknowledgements. This work has been completed within the Swiss Competence Center on Energy Research - Supply of Electricity, with support of Innosuisse and the Swiss National Science Foundation in the framework of the National Research Program 70 "Energy Turnaround".

Financial support. This research has been supported by the Swiss National Science Foundation (grant no. 407040_153889).

Review statement. This paper was edited by Michal Malinowski and reviewed by three anonymous referees.

\section{References}

Bakku, S. K., Fehler, M., and Burns, D.: Fracture compliance estimation using borehole tube waves, Geophysics, 78, D249-D260, 2013.

Barbosa, N. D., Caspari, E., Rubino, J. G., Greenwood, A., Baron, L., and Holliger, K.: Estimation of fracture compliance from attenuation and velocity analysis of full-waveform sonic log data, J. Geophys. Res.-Sol. Ea., 124, 2738-2761, 2019.

Bayes, T.: LII. An essay towards solving a problem in the doctrine of chances. By the late Rev. Mr. Bayes, F. R. $\mathrm{S}$. communicated by Mr. Price, in a letter to John Canton, A. M. F. R. S, Philosophical Transactions, 53, 370-418, https://doi.org/10.1098/rstl.1763.0053, 1763.

Chang, S. K., Liu, H. L., and Johnson, D. L.: Low-frequency tube waves in permeable rocks, Geophysics, 53, 519-527, 1988.

Cheng, C. H. and Toksöz, M. N.: Elastic wave propagation in a fluid-filled borehole and synthetic acoustic logs, Geophysics, 46, 1042-1053, 1981.

Coates, R. T.: A modelling study of open-hole single-well seismic imaging, Geophys. Prospect., 46, 153-175, 1998.

Daley, T. M., Gritto, R., Majer, E. L., and West, P.: Tube-wave suppression in single-well seismic acquisition, Geophysics, 68, 863869,2003

Falk, J., Tessmer, E., and Gajewski, D.: Tube Wave Modeling by the Finite-difference Method with Varying Grid Spacing, in: Pšenčík, I., Červený, V., and Klimeš, L. (Eds.): Seismic Waves in Laterally Inhomogeneous Media: Part 1, Pageoph Topical Volumes, Birkhäuser Basel, 77-93, 1996.

Gelman, A. G. and Rubin, D. B.: Inference from iterative simulation using multiple sequences, Stat. Sci., 7, 457-472, 1992.

Greenwood, A., Caspari, E., Egli, D., Baron, L., Zahner, T., Hunziker, J., and Holliger, K.: Characterization and imaging of a hydrothermally active near-vertical fault zone in crystalline rocks based on hydrophone VSP data, Tectonophysics, 750, 153-176, 2019a.

Greenwood, A., Dupuis, J. C., Kepic, A., and Urosevic, M.: Experimental testing of semirigid corrugated baffles for the suppression of tube waves in vertical seismic profile data, Geophysics, 84, D131-D149, 2019b.

Hardage, B. A.: An examination of tube wave noise in vertical seismic profiling data, Geophysics, 46, 892-903, 1981.

Hardin, E. and Toksöz, M. N.: Detection and characterization of fractures from generation of tube waves, Earth Resources Laboratory Industry Consortia Annual Report, Massachusetts Institute of Technology, 1985.

Herman, G. C., Milligan, P. A., Dong, Q., and Rector, J. W.: Analysis and removal of multiply scattered tube waves, Geophysics, 65, 745-754, 2000.

Hornby, B. E., Johnson, D. L., Winkler, K. W., and Plumb, R. A.: Fracture evaluation using reflected Stonely-wave arrivals, Geophysics, 54, 1274-1288, 1989.

Hunziker, J., Laloy, E., and Linde, N.: Bayesian full-waveform tomography with application to crosshole ground penetrating radar data, Geophys. J. Int., 218, 913-931, 2019.

Hunziker, J.: A semi-analytical forward solver to calculate VSP tube-wave data, GitHub, available at: https://github. com/rockphysicsUNIL/tube_wave_forward_solver, last access: 21 April 2020.

Karpfinger, F., Jocker, J., and Prioul, R.: Theoretical estimate of the tube-wave modulus in arbitrarily anisotropic media: Comparisons between semianalytical, FEM, and approximate solutions, Geophysics, 77, D199-D208, 2012.

Krietsch, H., Doetsch, J., Dutler, N., Jalali, M., Gischig, V., Loew, S., and Amann, F.: Comprehensive geological dataset describing a crystalline rock mass for hydraulic stimulation experiments, Sci. Data, 5, 180269, https://doi.org/10.1038/sdata.2018.269, 2018.

Kurkjian, A. L., Coates, R. T., White, J. E., and Schmidt, H.: Finite-difference and frequency-wavenumber modeling of seismic monopole sources and receivers in fluid-filled boreholes, Geophysics, 59, 1053-1064, 1994.

Laloy, E. and Vrugt, J. A.: High-dimensional posterior exploration of hydrologic models using multiple-try $\operatorname{DREAM}_{(\mathrm{ZS})}$ and high-performance computing, Water Resour. Res., 48, WO1526, https://doi.org/10.1029/2011WR010608, 2012.

Lee, J. M. and Toksöz, M. N.: Determination of the orientation of open fractures from hydrophone VSP, Earth Resources Laboratory Industry Consortia Annual Report, Massachusetts Institute of Technology, 1995.

Li, Y. D., Rabbel, W., and Wang, R.: Investigation of permeable fracture zones by tube-wave analysis, Geophys. J. Int., 116, 739753, 1994.

Metropolis, N., Rosenbluth, A. W., Rosenbluth, M. N., Teller, A. H., and Teller, E.: Equation of state calculations by 
fast computing machines, J. Chem. Phys., 21, 1087-1092, https://doi.org/10.1063/1.1699114, 1953.

Minato, S. and Ghose, R.: Low-frequency guided waves in a fluidfilled borehole: Simultaneous effects of generation and scattering due to multiple fractures, J. Appl. Phys., 121, 104902, https://doi.org/10.1063/1.4978250, 2017.

Minato, S., Ghose, R., Tsuji, T., Ikeda, M., and Onishi, K.: Hydraulic properties of closely spaced dipping open fractures intersecting a fluid-filled borehole derived from tube wave generation and scattering, J. Geophys. Res.-Sol. Ea., 122, 8003-8020, https://doi.org/10.1002/2017JB014681, 2017.

Nakagawa, S.: Low-frequency $(<100 \mathrm{~Hz})$ dynamic fracture compliance measurement in the laboratory, American Rock Mechanics Association, 2013.

Norris, A. N.: The speed of a tube wave, J. Acoust. Soc. Am., 87, 414-417, 1990.

Pyrak-Nolte, L. J.: Frequency dependence of fracture stiffness, Geophys. Res. Lett., 19, 325-328, 1992.

Schoenberg, M.: Elastic wave behavior across linear slip interfaces, J. Acoust. Soc. Am., 68, 1516-1521, 1980.

Sidler, R., Carcione, J. M., and Holliger, K.: A pseudo-spectral method for the simulation of poro-elastic seismic wave propagation in 2D polar coordinates using domain decomposition, J. Comput. Phys., 235, 846-864, 2013.
Tarantola, A.: Inverse Problem Theory and Methods for Model Parameter Estimation, Siam, https://doi.org/10.1137/1.9780898717921, 2005.

ter Braak, C. J. F. and Vrugt, J. A.: Differential Evolution Markov Chain with snooker updater and fewer chains, Stat. Comput., 18, 435-446, 2008.

Virieux, J. and Operto, S.: An overview of full-waveform inversion in exploration geophysics, Geophysics, 74, WCC127-WCC152, 2009.

Wenning, Q. C., Madonna, C., de Haller, A., and Burg, J.-P.: Permeability and seismic velocity anisotropy across a ductilebrittle fault zone in crystalline rock, Solid Earth, 9, 683-698, https://doi.org/10.5194/se-9-683-2018, 2018.

White, J.: Underground sound: Application of seismic waves, Elsevier, Amsterdam, 1983.

Zhu, Z., Chi, S., Zhan, X., and Toksöz, M. N.: Theoretical and Experimental Studies of Seismoelectric Conversions in Boreholes, Commun. Comput. Phys., 3, 109-120, 2008. 